\title{
Experience of managing first fifty COVID-19 positive peripartum women in a tertiary care centre in North India
}

\author{
Vijay Zutshi, Neha Mohit Bhagwati*, Alka
}

Department of Obstetrics and Gynecology, VMMC and Safdarjung Hospital, New Delhi, India

Received: 03 January 2021

Accepted: 06 February 2021

\section{*Correspondence:}

Dr. Neha Mohit Bhagwati,

E-mail: nehakwatra6188@gmail.com

Copyright: (c) the author(s), publisher and licensee Medip Academy. This is an open-access article distributed under the terms of the Creative Commons Attribution Non-Commercial License, which permits unrestricted non-commercial use, distribution, and reproduction in any medium, provided the original work is properly cited.

\begin{abstract}
Background: SARS-CoV-2 has caused significant morbidity and mortality worldwide. Analysis of the clinical profile of COVID-19 positive pregnant women is important to understand the pathophysiology, transmission and outcome of the disease in Indian population.

Methods: It is a retrospective observational study of first fifty pregnant patients tested positive for COVID-19 by qRT PCR admitted for delivery in our hospital.

Results: In this audit, first fifty COVID-19 pregnant women were studied and the mean age of the patients in this study was 26 years. $98 \%$ of these women were admitted for obstetric indications. Seventy two percent of these women were admitted with spontaneous onset of labour. Based on disease severity, 49 (98\%) were either asymptomatic or exhibited mild disease and only $1(2 \%)$ had severe disease who succumbed to her illness. Forty six percent patients delivered vaginally and 54\% required cesarean delivery. The most common indication for LSCS was fetal distress (43\%). Eighteen percent had preterm delivery. Among the newborn babies, one died due to severe birth asphyxia. Sixteen percent babies required NICU stay. Five babies tested positive for COVID-19 of which one baby was positive on day one of life.

Conclusions: As per our results, majority of the COVID-19 positive pregnant women had mild disease. There has been increase in cesarean section rate as compared to the previous hospital figures. Only one baby tested positive within 24 hours of delivery so the possibility vertical transmission can not be commented upon as of today.
\end{abstract}

Keywords: COVID-19, Corona virus, Maternal morbidity, Neonatal transmission, Pandemic

\section{INTRODUCTION}

Corona virus disease was detected in Wuhan, China and was first reported to the WHO on 31 Dec 2019. It was declared as a public health emergency of International concern on 30 Jan 2020. On 11 Feb 2020, WHO announced name and characteristics of a new coronavirus disease -"COVID-19". By March 11, WHO declared the COVID-19 a pandemic disease. ${ }^{1}$ By the time, authors are writing this paper, the pandemic has spread across 189 countries of the world with almost 42 million cases and over 1.15 million deaths worldwide. India has almost 7.8 million cases with 118,000 deaths. ${ }^{2}$
The International Committee on Taxonomy of Viruses has proposed severe acute respiratory syndrome coronavirus 2 (SARS-CoV-2) as the name of the virus that causes COVID-19. ${ }^{3}$ Coronaviruses belongs to the family Coronoviridae, order Nidovirales and are enveloped, non segmented, positive-sense ribonucleic acid (RNA) virus. ${ }^{4}$ There have been two other similar virus causing significant outbreak in last 2 decades, causing middle east respiratory syndrome (MERS) and severe acute respiratory syndrome (SARS). Clinical manifestations of COVID-19 are similar to those with SARS and MERS. 
Pregnancy is an immunologically altered state with physiological changes which could make pregnant women more susceptible to COVID-19 infection and it's complications. The issue of maternal management and fetal safety is a concern due to scarcity of data and guidelines on assessment and management of infected pregnant women, and a potential risk of vertical transmission. ${ }^{5}$ In the 2003 outbreak of SARS in Hong Kong, a series of SARS cases among pregnant women showed a case fatality of $25 \%$ with significant morbidity, mortality and adverse obstetric outcomes. ${ }^{6}$ As the clinical features of COVID-19 are similar to that of MERS and SARS, we need to be very careful regarding prevention and clinical management of COVID-19 in pregnant women.

\section{METHODS}

A retrospective review of COVID-19 positive pregnant patients admitted for delivery in the labor unit was done. The first COVID-19 case of a pregnant patient at our institution was detected on April $22^{\text {nd }} 2020$.

From the pregnant women coming to the hospital for various reasons and who were admitted, following information was collected at the designated triage counter located at the entrance of emergency receiving room; A detailed travel history, history of exposure to people with symptoms of COVID-19, symptoms of COVID-19, coming from hotspot area, immunocompromised condition.

Pregnant women meeting the criteria for COVID-19 screening were considered suspected cases until classified as negative and were admitted to specific designated labour unit for the same. Sampling was done in accordance with Indian council of medical research (ICMR) guidelines on patients with h/o symptoms suggestive of COVID-19 infection, history of travel to a place with COVID-19 outbreak, history of contact with laboratory confirmed case, residing in declared hotspot area (cluster/containment areas/large migration gatherings/evacuees center). ${ }^{7}$ Written informed consent was taken for testing of biological samples and neonatal pharyngeal swab samples.

A laboratory confirmed case was defined as a positive result on quantitative - reverse transcriptase- PCR (q RTPCR) assay of maternal nasal and oropharyngeal swab specimen. Complete epidemiological history, clinical symptoms and signs, laboratory and radiological findings, treatment measures and outcome data was recorded. Laboratory and radiological testing was done in accordance to clinical care need of the patient, individualized on basis of severity of symptoms. Date of onset of disease was taken as the date when symptoms were first noticed by the patient or the date of collection of sample in case of asymptomatic patient. Obstetric management of the COVID-19 patient was done as per standard practicing guidelines. Rooming in of all stable babies was done with clear instructions to the mother about hand hygiene and maternal masking. All neonates were subsequently tested for COVID-19 by qRT-PCR within 24 hours of delivery for babies born to laboratory confirmed COVID-19 mothers and at the earliest for babies whose mothers (COVID-19 suspects) were tested positive post delivery.

\section{Statistical analysis}

Categorical variables were presented in number and percentage (\%) and continuous variables will be presented as mean $\pm \mathrm{SD}$ and median. The data will be entered in MS EXCEL spreadsheet and analysis will be done using Statistical Package for Social Sciences (SPSS) version 21.0.

\section{RESULTS}

A total of 50 peripartum women admitted for delivery from April $22^{\text {nd }}$ to June $16^{\text {th }}$ who tested positive for COVID-19 infection were analysed. Among these, four patients were laboratory confirmed positive cases at the time of admission who were referred from other non COVID-19 hospitals in view of their positive COVID-19 report. All four patients were otherwise asymptomatic and were admitted for obstetric reasons. Forty six patients were suspected on the basis of symptoms $(n=37,74 \%)$, history of exposure to a COVID-19 positive person and symptoms $(n=1,2 \%)$, only history of exposure $(n=1,2 \%)$, history of residing in containment zone and symptoms $(\mathrm{n}=6,12 \%)$ and history of containment zone resident $(\mathrm{n}=1,2 \%)$.

Table 1: Distribution of socio-demographic characteristics of study subjects.

\begin{tabular}{|lll|}
$\begin{array}{l}\text { Socio-demographic } \\
\text { characteristics }\end{array}$ & Frequency & $\begin{array}{l}\text { Percentage } \\
(\%)\end{array}$ \\
\hline Age(years) & & \\
\hline$\leq 20$ & 5 & 10.00 \\
\hline $21-25$ & 21 & 42.00 \\
\hline $26-30$ & 18 & 36.00 \\
\hline$>30$ & 6 & 12.00 \\
\hline Mean \pm STDEV & $26.12 \pm 4.1$ & \\
\hline Median (IQR) & $25(23.25-28)$ \\
\hline Range & $19-38$ \\
\hline Parity & \\
\hline Primi gravida & 23 & \\
\hline Multigravida & 27 & 56.00 \\
\hline Period of gestation (weeks) & 54.00 \\
\hline$<31^{+6}$ & 1 & 2 \\
\hline $32-33^{+6}$ & 1 & 2 \\
\hline $34-36^{+6}$ & 7 & 14 \\
\hline $37-40$ & 34 & 68 \\
\hline $40^{+1}$ & 7 & 14 \\
\hline Mean \pm STDEV & $38.21 \pm 2.08$ \\
\hline Median(IQR) & $38.43(37.321-39.714)$ \\
\hline Range & $30.43-41.86$ \\
\hline
\end{tabular}


Of these 50 patients under study, 48 patients (98\%) were admitted for obstetric indications. Out of 48 patients, thirty six patients $(72 \%)$ were in spontaneous labour, induction of labour was done in 7 patients and elective cesarean section was done in 5 Patients. Induction of labor was done for severe preeclampsia $(n=1,2 \%)$, postdatism with severe oligohydramnios $(n=1,2 \%)$, postdatism with decreased fetal movements $(n=1,2 \%)$ and prolonged PROM $(n=3,6 \%)$. Of the 5 patients who underwent elective LSCS, two had developed symptoms post delivery, for two patients, elective LSCS was done only when they tested negative on repeat testing. For one patient, LSCS was done for CPD with obstetric cholesterols at 39 weeks of gestation after 7 days of testing. In this patient we did not wait for negative report with an opinion that prolongation could harm the foetus and an expectation of decreased viremia after one week of testing in an otherwise asymptotic patient.

Two patients were antenatal on admission. One of the patients was term pregnancy with history of previous 3 LSCS and was planned for elective cesarean section after 1 week and the second was a gravida 2 with rheumatic heart disease awaiting spontaneous labor. Both of these patients were discharged in stable condition with advice for isolation at home and follow up. The patients choose to deliver at some other centre and were lost to follow up.

The mean age of patients was 26 years. Twenty three patients $(46.00 \%)$ were primigravida and 27 patients $(54.00 \%)$ were multigravida. The mean gestational age at presentation to the hospital for delivery was 38 weeks. Nine patients $(18.00 \%)$ had preterm delivery before 37 completed weeks of gestation (Table 1). Eight of these patients had spontaneous preterm labour and one patient underwent Emergency LSCS in view of previous cesarean with severe FGR and fetal distress.

Eighty eight percent of patients were symptomatic. Fever was the most common symptom $(86.00 \%)$ followed by malaise (56\%) and cough (24\%) (Figure 1). Fever in most cases was low grade $\left(98.6-100.4^{\circ} \mathrm{F}\right)$ and intermittent. Seven patients developed symptoms before delivery whereas $7(14 \%)$ patients developed symptoms within one week of delivery. The mean duration from onset of symptoms to delivery was 2 days.

Nineteen patients (36\%) had additional co-morbid conditions with anemia $(n=31,62 \%)$ being the most common. Other co-morbid conditions included, hypothyroidism $(\mathrm{n}=6,12 \%)$, gestational hypertension $(n=3,6 \%)$, pre-eclampsia $(n=3,6 \%)$, asthma $(n=2,4 \%)$, gestational diabetes mellitus $(n=1,2 \%)$ and heart disease $(\mathrm{n}=1,2 \%)$. Patients with co-morbidities did not have any complications as compared to patients without comorbidites.

Based on COVID-19 disease severity characteristics by $\mathrm{ICMR}^{8}, 49(98 \%)$ were either asymptomatic or exhibited mild disease and only $1(2 \%)$ had severe disease who succumbed to her illness (Figure 2).

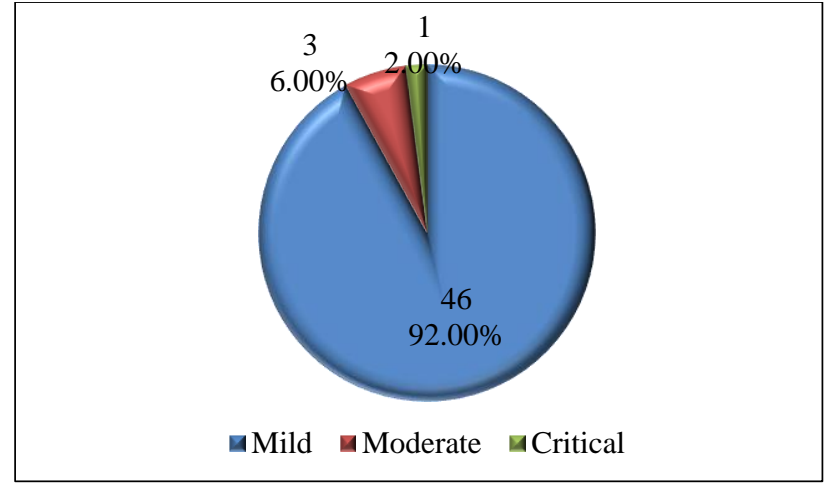

Figure 1: Distribution of disease severity of study subjects.

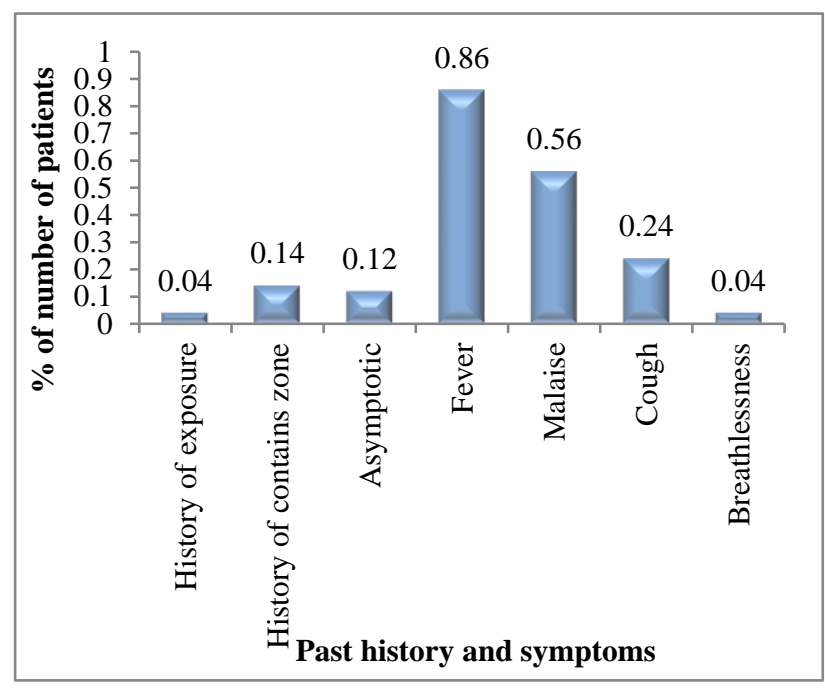

Figure 2: Distribution of past history and symptoms of study subjects.

Thirty one (62\%) women had anemia. The mean hemoglobin was $10.38 \mathrm{gm} / \mathrm{dl}$. The mean $\mathrm{N} / \mathrm{L}$ ratio was 2.82 (Table 2).

Forty six percent of patients delivered vaginally whereas $54 \%$ patients had undergone LSCS. The most common indication for LSCS was fetal distress (43\%) followed by previous LSCS in $32 \%$ cases (Figure 3). Overall fetal distress diagnosed during intrapartum electronic fetal monitoring was seen in $32 \%$ of patients. Fourteen of these patients had LSCS delivery and two delivered vaginally with perinatal asphyxia in baby evident by low APGAR score and need for resuscitation.

Among the newborn babies, there were total 49 babies as there was one twin delivery. Forty three babies had uneventful delivery and were shifted mother side. Eight babies required NICU stay (Table 3). The most common indication for NICU admission was low birth weight due to prematurity in 4 babies followed by birth asphyxia in three babies and GCA in one baby. One neonate died due to severe birth asphyxia. Five babies tested positive for COVID-19 of which one baby was positive on day one of 
life whose mother was a confirmed case before delivery. One was tested on day 3 and found positive, 2 babies on day 4 and found positive and one baby tested positive on day 10 of life after their mothers were diagnosed as
COVID-19 positive. These 4 mothers developed COVID19 specific symptoms in the post delivery period. None of these babies required any additional treatment or specific management and remained stable.

Table 2: Descriptive statistics of laboratory parameters of study subjects.

\begin{tabular}{|llll|}
\hline Laboratory parameters & Mean \pm STDEV & Median (IQR) & Range \\
\hline Hemoglobin $(\mathbf{g m} / \mathbf{d l})$ & $10.38 \pm 1.66$ & $10.3(9.8-11.35)$ & $6.8-14.7$ \\
\hline Total leucocyte count & $11426 \pm 4902.05$ & $10600(8025-13975)$ & $3300-24000$ \\
\hline N/L ratio & $2.82 \pm 0.87$ & $2.82(2.348-3.238)$ & $0.45-5.31$ \\
\hline Platelet count (in lakhs) & $5.55 \pm 26.34$ & $1.79(1.265-2.188)$ & $0.34-188$ \\
\hline
\end{tabular}

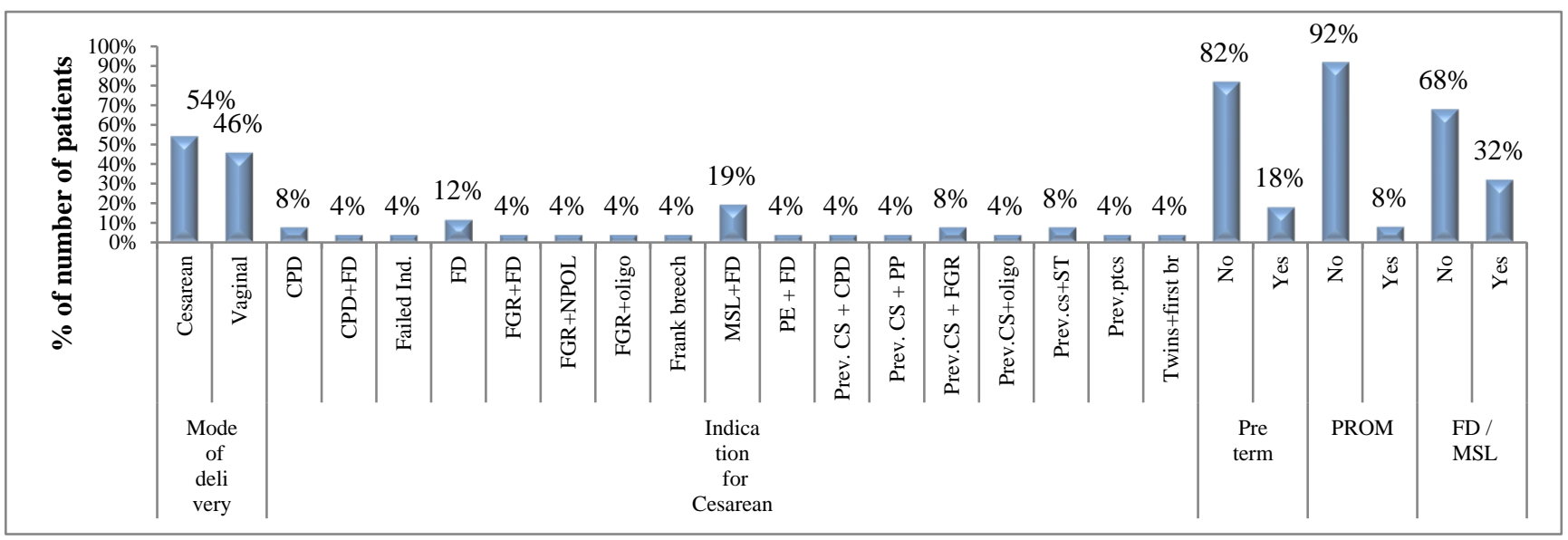

Figure 3: Distribution of maternal outcome of study subjects.

Table 3: Distribution of perinatal outcome of study subjects.

\begin{tabular}{|c|c|c|}
\hline Perinatal outcome & Frequency & Percentage \\
\hline \multicolumn{3}{|l|}{ NICU stay } \\
\hline No & 40 & 83.33 \\
\hline Yes & 8 & 16.67 \\
\hline \multicolumn{3}{|c|}{ Reason of NICU stay } \\
\hline Died & 1 & 12.50 \\
\hline FD & 2 & 25.00 \\
\hline GCA & 1 & 12.50 \\
\hline LBW & 4 & 50.00 \\
\hline \multicolumn{3}{|l|}{ Baby's COVID test } \\
\hline Negative & 43 & 89.58 \\
\hline Positive & 5 & 10.42 \\
\hline \multicolumn{3}{|l|}{ Baby weight(kg) } \\
\hline Mean \pm STDEV & $2.76 \pm 0.71$ & \\
\hline Median(IQR) & $2.75(2.4-3.1)$ & \\
\hline Range & $1.16-4.8$ & \\
\hline \multicolumn{3}{|c|}{ APGAR score at 1 minute } \\
\hline Mean \pm STDEV & $7.65 \pm 0.79$ & \\
\hline Median(IQR) & $8(7-8)$ & \\
\hline Range & $4-9$ & \\
\hline \multicolumn{3}{|c|}{ APGAR score at 5 minutes } \\
\hline Mean \pm STDEV & $8.77 \pm 0.66$ & \\
\hline Median(IQR) & $9(9-9)$ & \\
\hline Range & $6-10$ & \\
\hline \multicolumn{3}{|c|}{ Day of positive COVID test } \\
\hline Mean \pm STDEV & $4.8 \pm 4.21$ & \\
\hline
\end{tabular}




\begin{tabular}{|lll|}
\hline Perinatal outcome & Frequency & Percentage \\
\hline Median(IQR) & $4(3-4)$ & \\
\hline Range & $1-12$ & \\
\hline
\end{tabular}

\section{DISCUSSION}

Our hospital accounts for $7 \%$ of all births in the capital city of India, New Delhi with around 30,000 deliveries annually. ${ }^{14}$ As the number of COVID-19 cases started to increase, a separate obstetric isolation unit including a labour room and an operation theatre was created for laboratory confirmed and suspected COVID-19 patients. This helped not only in prevention of cross infection amongst patients but also protection of doctors and hospital staff from getting infected. This study was done during the initial nationwide lockdown.

\section{Disease course and severity}

Spread of COVID-19 infection has affected pregnant women worldwide. Although pregnancy is an immunosuppressive state which makes pregnant women more susceptible to infections but as most of these women are young and have less co-morbidities and better health status, they seem to have mild disease. As per our results, the mean age of patients was 26 years with mild disease in $98 \%$ cases. Various studies done recently by Breslin et al, Qiancheng et al and Zaigham et al where 43, 28 and 108 patients were analyzed respectively, also showed mild to moderate disease in majority of patients. ${ }^{9-}$ 11 Breslin et al in their study on 43 patients have documented mild disease in $86 \%$ cases. $^{9}$ In these studies maximum number of patients were young with mean age of 30 which is similar to our results. ${ }^{9-11}$ The median gestational age on admission was 38 weeks which is similar to the retrospective studies by Yan et al in China and Breslin et al in New York. ${ }^{9,12}$ In our study, $12 \%$ patients were asymptomatic and were tested due to epidemiological reasons. Because in the beginning of the lockdown the guidelines were still evolving and there was no clarity on the testing protocols and also the number of patients in our study is small. On the contrary, Yan et al reported $23.3 \%$ asymptomatic cases which could probably be because of more testing done there. ${ }^{12}$ Ninety eight percent patients exhibited only mild symptoms throughout the course of the disease with fever being the most common symptom. Similar results have been seen in study done by Yan et al with fever been the most common presentation in $50.9 \%$ cases. ${ }^{12}$ Since majority of our patients had mild disease, we had only one mortality. Our patient who died had developed severe pneumonia and acute respiratory distress on post operative day two. No cases of severe pneumonia or maternal death was reported by Schwartz et al in their study on 38 women in China. ${ }^{13}$ During the previous SARS epidemic in 2013, Wong et al reported the case fatality rate was $25 \%$ among pregnant women. ${ }^{6}$ It seems that COVID-19 is not causing too many fatality. As per the available evidence, COVID-19 infection has a milder course as compared to previous SARS group infection epidemics. Since majority of these women exhibit mild disease so most of the admissions are done for obstetric reasons. Universal testing for patients being admitted for obstetric indications to hospitals is desirable so that they could be transferred to designated isolation COVID-19 facility to prevent cross infections to other patients and their neonates.

\section{Pregnancy complications and outcome}

The cesarean section rate in our institution has been fluctuating between $28-30 \%$ over the last three years. ${ }^{14}$ In our study, $54 \%$ patients had cesarean delivery with fetal distress as the most common indication (43\%). Overall, $32 \%$ patients had fetal distress during intrapartum period. The observation from these results seem to suggest that the cesarean rate in these patients has increased. Breslin $\mathrm{N}$ et al also reported the cesarean section rate as $44.4 \%$ with fetal distress being the most common indication $(37.4 \%) .{ }^{9}$ In the study by Yan et al, amongst 99 delivered COVID-19 positive patients, $85.9 \%$ had cesarean deliveries. Amongst these, $38.8 \%$ were done for pneumonia followed by previous cesarean section $(18.8 \%)$ and fetal distress $(10.6 \%){ }^{12}$ These results are in contrast to our results and results by Breslin et al where the cesarean section done for fetal distress were much higher than this. ${ }^{9}$ Whether this increase in the cesarean section rate by $26 \%$ can be attributed to viremia needs to be further evaluated. The rate of preterm birth in our study was $18 \%$ of which $88 \%$ had spontaneous preterm labour. This is significantly more than the average preterm birth in our country which is $13.6 \% .{ }^{15}$ On the contrary, Yan et al showed that twenty one percent patients had preterm birth with $6.1 \%$ had spontaneous preterm labour. ${ }^{12}$ This again emphasize possible high viral load as the probable cause of preterm deliveries.

\section{Neonatal outcome}

Our study result shows that fetal distress was the most common indication for cesarean section but only 3 babies required NICU stay. This probably could be explained that fetal distress was an acute event and timely intervention avoided birth asphyxia. Our study results show that eight out of 51 babies were admitted in NICU with prematurity as the most common indication followed by birth asphyxia. There was one neonatal death due to severe birth asphyxia. Similar result was shown by Yan et al where only one neonatal death occurred due to severe birth asphyxia. ${ }^{12}$ One baby born by vaginal route tested positive for the infection on day 1 of life and this mother had mild symptoms and was in spontaneous labour with adequate progression of labour and uneventful delivery. It is difficult to extrapolate the mode of transmission as to either vaginal secretions or transplacental. Whereas the other 4 babies who tested positive on day $3,4,4$ and 12 do not qualify to assess the mode of transmission. Their postnatal testing was delayed 
due to which their COVID-19 positive status cannot be completely attributed to vertical mode of transmission. Direct transmission through the mothers or environmental exposure cannot be ruled out in them. The baby born to the mother who died after developing severe pneumonia and ARDS tested negative for the infection and remained asymptomatic. All these babies were asymptomatic, were kept under vigilant observation and had an uneventful postnatal stay. Yan et al showed no evidence of vertical transmission in their study in 100 neonates who were tested. ${ }^{12}$ In contrast to this, Zheng et al, in a study series of 33 neonates documented that 3 neonates were symptomatic for COVID-19 with positive RT-PCR in anal and nasopharyngeal swabs. ${ }^{16}$ And $\mathrm{Yu}$ et al, one newborn delivered by cesarean section with no contact with the mother, had a positive RT-PCR in pharyngeal swan collected 36 hours after birth. ${ }^{17}$

\section{CONCLUSION}

In conclusion, COVID-19 infection in pregnant women does not increase the morbidity as the patients are relatively young. The rate of fetal distress necessitating emergency cesarean delivery has been seen. So we suggest that these patients must therefore be kept under continuous intrapartum electronic survellience and prompt decision for cesarean section should be taken whenever indicated to prevent occurrence of birth asphyxia. There is no clarity as of now about the vertical transmission to neonates. The limitation of the study is the small sample size and further studies will give us more insight in future.

\section{Funding: No funding sources}

Conflict of interest: None declared

Ethical approval: The study was approved by the Institutional Ethics Committee

\section{REFERENCES}

1. Cascella M, Rajnik M, Cuomo A, et al. Features, Evaluation and Treatment Coronavirus (COVID-19). In: StatPearls. Treasure Island (FL): StatPearls Publishing; 2020.

2. Max Roser, Hannah Ritchie, Esteban Ortiz-Ospina and Joe Hasell (2020) - "Coronavirus Pandemic (COVID-19)". Available at: OurWorldInData.org. https://ourworldindata.org/coronavirus'.

3. Coronaviridae Study Group of the International Committee on Taxonomy of Viruses. The Severe acute respiratory syndrome-related coronavirus: classifying 2019-nCoV and naming it SARS-CoV-2. Nat Microbiol. 2020;5(4):536-44.

4. Su S, Wong G, Shi W, Liu J, Lai AC, Zhou J, et al. Epidemiology, Genetic Recombination, and Pathogenesis of Coronaviruses. Trends Microbiol. 2016;24(6):490-502.

5. Luo Y, Yin K. Management of pregnant women infected with COVID-19. Lancet Infect Dis. 2020;20(5):513-4.
6. Wong SF, Chow KM, Leung TN, Ng WF, Ng TK, Shek CC, et al. Pregnancy and perinatal outcomes of women with severe acute respiratory syndrome. Am J Obstet Gynecol. 2004;191(1):292-7.

7. Indian council of medical research. Revised Strategy for COVID-19 testing in India. Available from: https://www.mohfw.gov.in/pdf/Revisedtestingguideli nes.pdf. Accessed on 18 May 2020.

8. Government of India Ministry of Health and Family Welfare Directorate General of Health services (EMR division). Clinical Management Protocol for COVID-19. Available from: https://www.mohfw.gov.in/pdf/ClinicalManagement ProtocolforCOVID19.pdf. Accessed 13 June 2020.

9. Noelle B, Caitlin B, Cynthia GB, Russell M, Rebecca M, Kyra B, et al. COVID-19 infection among asymptomatic and symptomatic pregnant women: Two weeks of confirmed presentations to an affiliated pair of New York City hospitals. Am J Obstet Gynecol MFM. 2020:100118.

10. Qiancheng X, Jian S, Lingling P, Lei H, Xiaogan J, Weihua L, et al. Coronavirus disease 2019 in pregnancy. Int J Infect Dis. 2020;95:376-83.

11. Zaigham M, Andersson O. Maternal and perinatal outcomes with COVID-19: A systematic review of 108 pregnancies. Acta Obstet Gynecol Scand. 2020;99(7):823-9.

12. Yan J, Guo J, Fan C, Juan J, Yu X, Li J, et al. Coronavirus disease 2019 (COVID-19) in pregnant women: A report based on 116 cases. Am J Obstet Gynecol. 2020;223(1):111-e1.

13. Schwartz DA. An Analysis of 38 Pregnant Women with COVID-19, Their Newborn Infants, and Maternal-Fetal Transmission of SARS-CoV-2: Maternal Coronavirus Infections and Pregnancy Outcomes. Arch Pathol Lab Med. 2020;144(7):799805 .

14. Mittal P, Pandey D, Suri J, Bharti R. Trend Prediction for Cesarean Deliveries Based on Robson Classification System at a Tertiary Referral Unit of North India. J Obstet Gynaecol India. 2020;70(2):111-8.

15. Chawanpaiboon S, Vogel JP, Moller AB, Lumbiganon P, Petzold M, Hogan D, et al. Global, regional, and national estimates of levels of preterm birth in 2014: a systematic review and modelling analysis. Lancet Glob Health. 2019;7(1):e37-e4.

16. Zeng L, Xia S, Yuan W, Yan K, Xiao F, Shao J, et al. Neonatal early-onset infection with SARS-CoV-2 in 33 neonates born to mothers with COVID-19 in Wuhan, China. JAMA pediatrics. 2020;174(7):722-5.

17. Yu N, Li W, Kang Q, Xiong Z, Wang S, Lin X, et al. Clinical features and obstetric and neonatal outcomes of pregnant patients with COVID-19 in Wuhan, China: a retrospective, single-centre, descriptive study. Lancet Infec Dis. 2020;20(5):559-64.

Cite this article as: Zutshi V, Bhagwati NM, Alka. Experience of managing first fifty COVID-19 positive peripartum women in a tertiary care centre in North India. Int J Reprod Contracept Obstet Gynecol 2021;10:1036-41. 